\title{
The Concept of Evolution of Thoracolumbar Fracture Classifications Helps in Surgical Decisions
}

\author{
Md Quamar Azam, Mir Sadat-Ali \\ Department of Orthopedics, College of Medicine, University of Dammam, Dammam, Saudi Arabia
}

Since Boehler's sentinel description, a universally acceptable thoracolumber fracture classification has eluded spine surgeons. The concept of the stability of a thoracolumbar injury changed continuously from the two column concept of Holdsworth to the three column theory of Denis. With the advent of sophisticated imaging techniques, improved biomechanical understanding, and in order to meet the high expectations of patients, several classification systems have been forwarded by the stalwarts in the field. Each successive system has contributed significantly to the understanding and prediction of treatment outcome. Load sharing classification by McCormack attempted to rationalize the use of short segment posterior instrumentation. Magerl et al. developed a comprehensive classification system based on progressive morphological damage determined by three fundamental forces: compression, distraction, and axial torque. Vaccaro et al. devised the thoracolumbar injury severity score based on three independent variables: the morphology of the injury, posterior ligamentous complex (PLC) integrity, and neurological status at the time of injury. But, there are limitations to the classification system, especially when magnetic resonance imaging yields a PLC status as indeterminant. In the absence of a universally acceptable classification system, it is important to understand the underlying concepts of the fractures. The author concisely reviews the subject from its inception in the year 1929 to the present day.

Keywords: Evolution; Thoraco-lumbar; Fracture; Stability

\section{Introduction}

For more than eight decades, since Boehler's [1] sentinel description of the fracture, a universally acceptable thoracolumbar fracture classification has eluded trauma and spine surgeons. Constant efforts by the experts in the field with the support of the new sophisticated and better imaging modalities has improved our understanding of the fractures. However, in the past few decades, with an increase in complex accidents either on the roads or elsewhere (industrial, occupational, skiing) along with the high demand and expectations of patients, has continued to pose challenges.

The severity of an injury is a continuous event. Therefore, drawing any arbitrary division is bound to create a gray zone where treatment modalities like conservative vs. surgery, posterior vs. anterior instrumentation, and whether to fuse or not will remain subjects of discussion. A classification system is required to facilitate effective communication between spine surgeons, to guide treatment, and to help predict the prognosis. An ideal system should be simple, comprehensive, reliable, and reproducible with predictive outcomes. Unfortunately, most of the existing classifications have failed to fulfill the above crite-

\footnotetext{
Received Apr 2, 2015; Accepted Apr 26, 2015

Corresponding author:Md Quamar Azam

Department of Orthopedics, KFHU, University of Dammam, PO Box- 40071, Al-Khobar-31952, Saudi Arabia

Tel: +966-532750228, E-mail: qazam47@gmail.com
} 
ria; some are oversimplified while others are too inclusive and complex for routine use. Given the scenario where none of the classification is accepted worldwide, it is imperative to understand the evolution of spinal injury classification. The authors concisely review the subject from its inception in the year 1929 to the present day.

\section{Biomechanics of the Thoracolumbar Spine}

The thoraco-lumbar (TL) spine essentially consists of the T10 to L2 region, which is the most common site afflicted by trauma, L1 being the most common vertebra, followed by T12 [2-6]. Its predilection and vulnerability for injury stems from the following reasons: (1) Mobility: the TL spine is one of the most mobile segments, and thus is subjected to all kinds of forces including compression, flexion, extension, and rotation, resulting in its vulnerability to fracture. (2) Transition zone: the TL region is relatively straight (kyphosis from $0-10$ degrees) and situated between the kyphotic thoracic and lordotic lumber spine. Unlike the thoracic spine, the absence of costo-vertebral structures no longer buttress the TL area. (3) Facet joints: Facet joints of the thoracic spine are coronally oriented to resist flexion-extension and that of lumbar spine is sagitally oriented to allow flexion-extension. In TL area facet joints shows a transition from predominantly coronal to predominantly sagital orientation.

\section{Boehler (1929) [1]}

Based on the mechanism of injury and geometry of fracture, Boehler set up a classification system for TL fractures for the first time. His classification included five categories: compression fracture, flexion distraction injury (compression injury to the vertebral body and distraction injury to the posterior element), extension injury, shear fracture, and rotational injuries.

\section{Watson-Jones (1938) [7]}

Watson Jones, in 1938, was the first to introduce the concept of stability into the classification and emphasized that the "integrity of the posterior ligamentous complex (PLC) is essential for stability". Based on 252 cases, the classification system described three types of vertebral fractures and later added fourth type: (1) simple wedge fracture, (2) comminuted fracture; (3) fracture dislocation, (4) hyper- extension injuries.

\section{Watson's concept (of stability)}

Watson observed that severe comminuted vertebral body fractures are not painful, while severe wedge fractures cause pain. He attributed this to the tremendous stress to the posterior intra-articular joints due to severe wedging anteriorly. He stressed that, if the radiological alignment is not improved, $30 \%-40 \%$ of these patients will develop moderate to severe pain. He went on to describe, in detail, the method and technique of application of casting in such cases. He further noted that, ruptures of discs in severely comminuted fractures lead to severe narrowing of disc space and bony fusion of the adjacent vertebrae by ankyloses, and hence, they are painless.

\section{Nicoll (1949) [8]}

Nicolle sought to validate the Watson-Jones thesis which stated that "anatomic reduction is indispensable for a good functional result". Based on 162 TL fractures, he explained that nucleus pulposus, and not the intra-articular joint, acts as a hinge in flexion injuries. So, if it remains intact, wedge fractures cause strain on the posterior ligaments. He advised that, in any injury, the following four structures should be examined separately: the vertebral body, disc, intra-articular joint, and the inter-spinous ligament.

He divided TL fractures into the following four categories: (1) Anterior wedging. (2) Lateral wedging (flexionrotation injury): often identified as a transverse process fracture on the convex side and damage to posterior inter-articular joint on the concave side. (3) Fracturedislocation: associated with a rupture of the inter-spinous ligament with fracture-dislocation of facet joints. (4) Neural arch injury: rotation injury with a fracture of the intraarticular part of the lamina.

\section{Nicoll's concept of stability}

Mild to moderate anterior wedging and lateral wedge fractures and all lamina fractures above the L4 level are stable and can be treated conservatively without any danger of deformity increasing and without a threat to the spinal cord. Severe wedge fractures and bilateral lamina fractures below L4 are essentially unstable injuries. 


\section{Sir Frank Holdsworth (1970) [9]}

After reviewing over 1000 patients at Sheffield Hospital in England, Holdsworth revolutionized thoracolumbar fracture classification by introducing the "Two Column Concept". He divided the spine into the anterior column, which is formed by vertebral body and the disc, and the posterior column, which consists of the facet joints and PLC. His classification scheme included the following: (1) Anterior compression injury. (2) Rotational fracture dislocation (flexion rotation injury). (3) Extension injury. (4) Shear injury. (5) Burst fracture (introduced for the first time).

\section{Holdsworth's concept of stability}

Sir Holdsworth emphasized the importance of the PLC in maintaining stability and stressed that the presence of a palpable gap posteriorly represents an unstable spine, even if $\mathrm{X}$-rays are equivocal. He labeled simple wedge fractures and burst fractures as inherently stable, deeming a few days to weeks of immobilization in a plaster jacket followed by mobilization as the appropriate course of treatment. In comminuted burst fractures, as the fragments are not impacted, longer immobilization is required, but fusion is almost always the rule. Pure dislocations even without fractures are, however, unstable as ligament healing is poor at best. In such cases, he recommended surgical fusion.

\section{Francis Denis (1983) [10]}

Based on retrospective analysis of 412 TL fractures, Denis introduced an abstract concept of a middle column that included the posterior wall of the vertebral body, posterior annulus fibrosus, and posterior longitudinal ligament (PLL). He propounded the three column concept of the spine (Fig. 1). The anterior column is formed by anterior longitudinal ligament (ALL), anterior annulus fibrosus, and the anterior part of vertebral body. The posterior col-

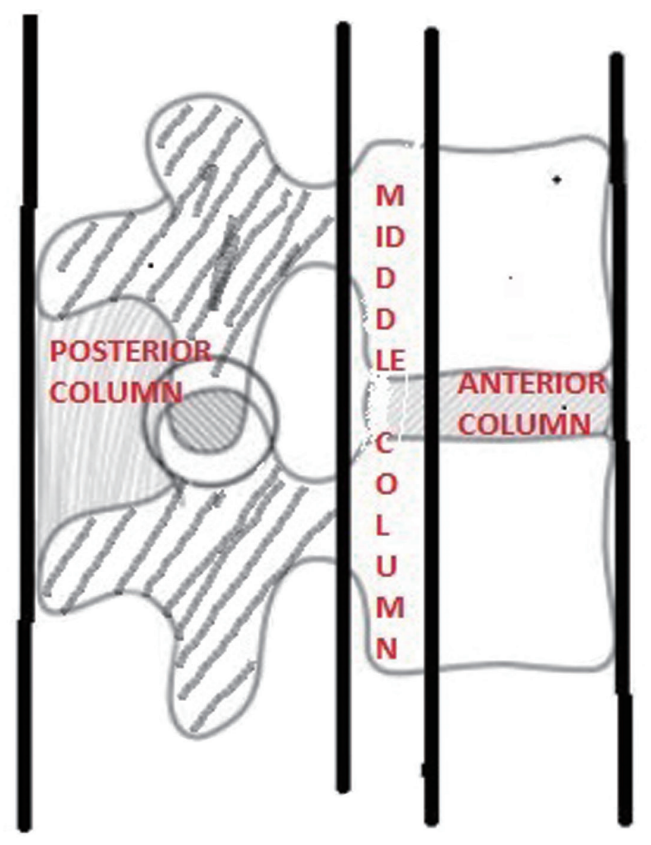

\begin{tabular}{|l|l|l|}
\hline Posterior column & Middle column & Anterior column \\
Posterior bony arch+ & Posterior longitudinal & Anterior longitudinal \\
supraspinous ligament+ & ligament+posterior & ligament+anterior \\
interspinous ligament+ & vertebral wall+ & annulus fibrosus + \\
ligamentum flavum & posterior annulus fibrosus & anterior vertebral body \\
\hline
\end{tabular}

Fig. 1. Denis Classification of major vertebral injuries: compression fractures, burst fractures, seat belt-type injuries, and fracture dislocations. 
Table 1. Column involvement in major Denis fracture types

\begin{tabular}{|c|c|c|c|}
\hline Denis major type & Anterior column & Middle column & Posterior column \\
\hline 1. Compression fracture & Compression fracture & None & None $^{\text {a) }}$ \\
\hline 2. Burst fracture ${ }^{b)}$ & Compression & Compression & None \\
\hline 3. Seat-belt-type injury ${ }^{b /}$ & None & Distraction & Distraction \\
\hline 4. Fracture dislocation & Compression \& rotation shear & Distraction \& rotation shear & Distraction \& rotation shear \\
\hline
\end{tabular}

${ }^{\text {a) }}$ Rarely in very severe compression fracture of anterior column distraction of posterior column may occur; ${ }^{\text {b) }}$ Both represent two column involvement, but in burst fracture anterior column is involved while in seat-belt-type injury posterior column is involved; middle column is common.

umn essentially remains the same as described by Holdsworth i.e., the posterior bony complex along with the PLC. The PLC consists of the supra-spinatous and intraspinous ligaments, ligamentum flavum, and the capsule of intra-articular joints. Denis differed from Holdsworth by maintaining that PLC rupture alone does not lead to instability unless there is involvement of the PLL, posterior vertebral body, and posterior annulus.

Spinal injuries were divided into minor injuries (fracture of transverse process, fracture of articular process, fracture of pars-intra-articularis, and isolated spinous fracture) and major injuries. Major injuries were classified into four categories, including: compression fracture, burst fracture, seat-belt-type injury, and fracture dislocation (Table 1). These were further subdivided into more than 20 subtypes as follows: (1) Compression fracture: the failure of the anterior column under compression. The middle column, being totally intact, acts as a hinge causing posterior column tension. So, in severe compression injuries, the posterior column is affected. (2) Burst fracture: the distinguishing feature is the failure of middle column under axial compression resulting in loss of posterior vertebral height, retropulsion of vertebral body fragment in neural canal with or without neurological involvement. The radiolograph is characterized by an increase in inter-pedicular distance, vertical fracture of the lamina, and the splaying of the posterior joint. (3) Seat-belt-type injury: represents the failure of both the posterior and middle columns under tension, and is characterized by the increased height of the posterior vertebral body and the opening of the disc space. A plain radiograph shows an increase in inter-spinous distance, horizontal split of the pedicle, and horizontal split of the transverse process. (4) Fracture-dislocation: characterized by the failure of all columns under compression, tension, rotation, or shear stress leading to sub-luxation or dislocation. It is divided in three types and respective sub-types: a) Flexion-rotation: the posterior and middle columns are totally ruptured under tension and rotation forces. The anterior column fails under a combination of compression and rotation forces leading to anterior wedging of the vertebral body, partial tear of the antero-lateral periosteum, and stripping of the ALL. Flexion-rotation injuries may go through the disc or through the vertebral body, known traditionally as the Slice fracture. b) Sheartype fracture dislocation: all three columns are disrupted most frequently by postero-anterior shear forces, but the dislocation can also be caused by antero-posterior forces. In the postero-anterior shear force, the segment above moves forward relative to the segment below and, in the process, the posterior arch of the superior vertebral body is fractured. In cases resulting from antero-posterior shear force, the superior segment is pushed backward without any fracture of the posterior arch. c) Flexion-distractiontype: resembles the seat-belt-type injury [11] where both the posterior and middle columns rupture with tension in addition to tearing of the entire annulus fibrosus. This allows the vertebral body above to subluxate or dislocate relative to the vertebral body below.

\section{Denis concept of stability}

(1) Stable fracture: minimal to moderate compression fracture represents a stable injury and may be treated by early ambulation with or without external immobilization.

(2) First degree instability (mechanical instability): represented by a severe compression fracture and seatbelt-type injury. In the former, the spine buckles around the intact middle column hinge whereas, in the latter, the spine buckles around the intact anterior column.

(3) Second degree instability (neurologic instability): 
burst fractures; because $20.3 \%$ of burst fracturse with intact neurology initially develop become neurologically compromised during follow-up.

(4) Third degree instability (mechanical \& neurological instability): represented by a severe burst fracture with neurological compromise and fracture-dislocation. Denis practiced and recommended surgical decompression and surgical stabilization in this group.

\section{Paul C McAfee (1983) [12]}

McAfee studied the computed tomography (CT) scans of 100 TL fractures, and combined the individual suggestions of Holdsworth [9], White and Panjabi [13], and Denis [10] classifications to develop a simpler classification based on the mechanism of injury and its morphology. $\mathrm{He}$ identified three forces; axial compression, axial distraction, and translation.

\section{McAfee's concept of stability}

(1) For the first time, the burst fracture was subdivided into two types of fracture: a stable burst fracture (anterior and middle column fails due to compression forces without involving the posterior column) and an unstable burst fracture (posterior column is involved due to compression/lateral flexion / rotation forces) illustrated by facet joint sub-luxation or disruption of the neural arc.

(2) McAfee also separated the seat-belt-type injury into the chance fracture (bony) and other flexion distraction injury with pure ligamentous involvement. The second group is unstable and usually requires surgery.

\section{McCormack (1994) [14]}

With the popularity of short segment pedicle screw (SSPS) construct, several shortcomings surfaced in the form of failure of fixation and progression of kyphosis. After retrospectively reviewing his cases, McCormack devised a scoring system to help assess the risk of failure of the SSPS construct. The group identified three factors that co-relate with failure of the posterior short segment pedicle screw system.

(1) Degree of kyphosis correction on lateral view

(2) Degree of vertebral comminution

(3) Apposition of fracture fragment

Each is graded as mild, moderate, or severe with cor- responding point values of 1,2 , and 3 , respectively, with a total score ranging from 3 to 9 (Fig. 2). The higher scores demonstrate weaker anterior column support.

\section{McCormack concept of stability}

(1) If the score is 6 or less, it represents a stable fracture and posterior SSPS fixation may be used.

(2) If the score is 7 or more without translation, an anterior surgical approach with anterior column support using an instrument and strut-graft is indicated.

(3) If the score is 7 or more with fracture-dislocation; staged surgery is advisable; short segment posterior fusion followed by anterior column support is recommend. Alternatively, long segment posterior instrument could be used, but it compromises mobility.

This classification system helps the surgeon to decide whether to use short segment fixation using a posterior or anterior approach depending on the comminution of vertebral body.

This load sharing classification has shown high degrees of inter- and intra-observer reliability [15]. However, as Scholl et al. [16] pointed out, this system does not incorporate ligamentous or neurological damage, and hence cannot be used to assess surgical indication.

\section{The AO (Arbeitsgemeinschaft fur Osteosynthesenfragen)/Magerl Classification (1994) [17]}

After a diligent decade-long research analysis of 1445 cases, Magerl et al. [17] developed a comprehensive classification of TL fractures. The system is based on progressive morphological damage determined by three fundamental forces; compression, distraction, and axial torque (rotation). This classification deems the middle column to be virtual and not an anatomic entity, and thus not fit to be used as a denominator of an injury type.

A simple grid 3-3-3 scheme of $\mathrm{AO}$ fracture classification is used. It consists of three types: A, B, and C (Fig. 3). Every type has three groups, each of which contains three subgroups with specifications. Severity progresses from type A through type $\mathrm{C}$ as well as within the subgroups. Type A comprises about $60 \%, \mathrm{~B}, 15 \%$, and C, $20 \%$. Overall neural injuries were noted in $14 \%$ of type A, $32 \%$ of type $B$, and $55 \%$ of type $C$. However, the percentage of neural injuries in A3 was 32\%, which is similar to B1 (30\%) and B2 


\begin{tabular}{|l|l|l|l|}
\hline & $\begin{array}{l}\text { Grade-1 } \\
\text { (mild) }\end{array}$ & $\begin{array}{l}\text { Grade-2 } \\
\text { (moderate) }\end{array}$ & $\begin{array}{l}\text { Grade-3 } \\
\text { (severe) }\end{array}$ \\
\hline $\begin{array}{l}\text { Comminution } \\
\text { (sagittal CT scan) }\end{array}$ & $<30 \%$ & $30 \%-60 \%$ & $>60 \%$ \\
\hline $\begin{array}{l}\text { Apposition of } \\
\text { fragment } \\
\text { (axial CT scan) }\end{array}$ & $\begin{array}{l}\text { Minimal } \\
\text { displacement }\end{array}$ & $\begin{array}{l}2 \mathrm{~mm} \\
\text { displacement } \\
<50 \% \text { cross section }\end{array}$ & $\begin{array}{l}2 \mathrm{~mm} \\
\text { displacement } \\
>50 \% \text { cross section }\end{array}$ \\
\hline $\begin{array}{l}\text { Kyphosis correction } \\
\text { (lateral X-ray) }\end{array}$ & $\leq 3^{\circ}$ or less & $4^{\circ}-9^{\circ}$ & $\geq 10^{\circ}$ \\
\hline
\end{tabular}

Calculate the score

\begin{tabular}{|l|l|}
\hline Score (3 to 9) & Suggested surgical plan \\
\hline$<6$ & Short segment posterior stabilization \\
\hline$\geq 7$ without translation & Anterior instrument and strut-graft \\
\hline$\geq 7$ with fracture-dislocation & $\begin{array}{l}\text { Staged surgery; posterior SSPS followed } \\
\text { by anterior column support. } \\
\text { or } \\
\text { Long segment posterior stabilization }\end{array}$ \\
\hline
\end{tabular}

Fig. 2. Fracture severity point values assigned by McCormack et al. [14] based on three factors: comminution, apposition, and kyphosis. CT, computed tomography; SSPS, short segment pedicle screw.

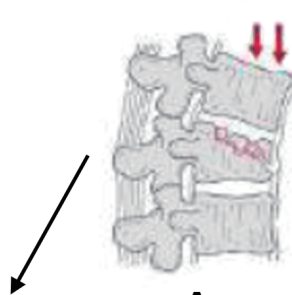

A

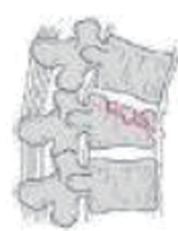

A1

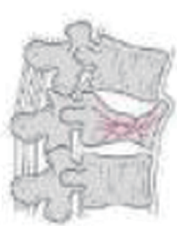

A2

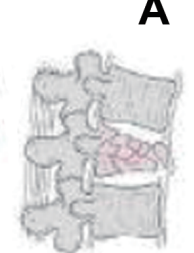

A3

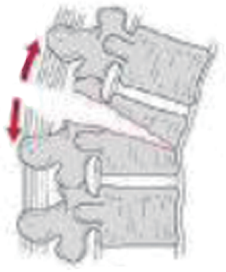

B

B

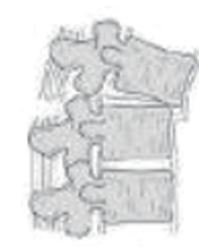

B1

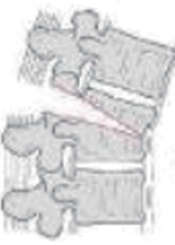

B2
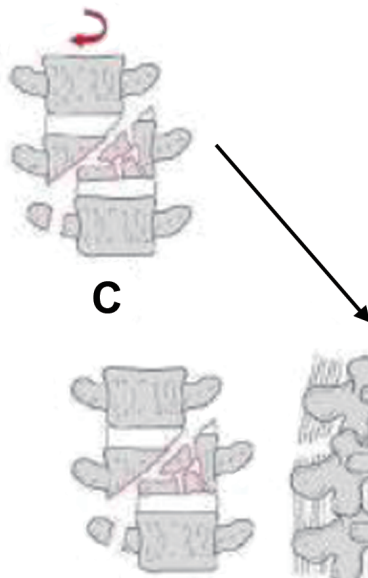

C1

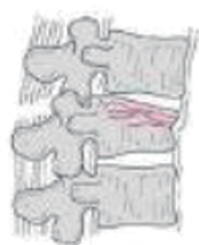

C2

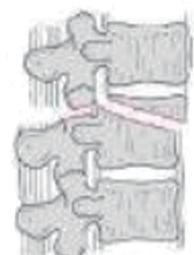

C3

Fig. 3. AO's (A) compression, $(B)$ distraction, and $(C)$ axial torque injury classifications. Each injury type is further subdivided into three groups. 
(33\%). Magerl et al's analysis found that the neurological involvement in C2 was more (60\%) than in C3 (50\%), and they attributed this to the smaller number of cases in C3.

\section{Type A}

Type A results from compressive forces resulting in vertebral body injuries in compression with insignificant posterior column injuries. It is clinically characterized by mild to moderate posterior tenderness without swelling or subcutaneous hematoma.

Radiographic characteristics:

(1) Loss of anterior vertebral height mostly (kyphosis) or posterior vertebral body fracture (burst),

(2) Vertical split of lamina accompanied by increased horizontal distance between pedicles, and

(3) Posterior wall fragments are displaced only posteriorly i.e., neither cranial nor translational.

\section{Type B}

Type B is a result of tensile forces which cause anterior and posterior element injuries with a distraction characterized by the transverse disruption either anteriorly or posteriorly. Injuries are subdivided into B1, characterized by transverse disruption of posterior ligament complex; and B2, the transverse disruption of posterior bony element. Both types result from distraction injuries when the center of rotation (COR) falls anterior to the vertebral body. B3 injuries are hyperextension injuries caused when the COR falls posterior to the vertebral body, leading to distraction and failure of the anterior column.

Clinically, type B injuries are characterized by marked tenderness, swelling, and subcutaneous hematoma posteriorly. A palpable gap between the spinous processes or a step is pathognomonic of $\mathrm{B} 1$ and $\mathrm{B} 2$ injuries.

Radiographic characteristics:

(1) Transverse disruption PLC alone as in B1, or of the posterior column (lamina and/or pedicle) as in B2,

(2) Significant increase in interspinous distance ( $>7$ $\mathrm{mm})$

(3) Anterior translational displacement,

(4) Facet joints showing bilateral sub-luxation/dislocation/fracture,

(5) Inverse cortical sign present; post wall fragment rotated up to 90 degrees around the transverse axis so that post smooth part faces forward. Also, the fragment may migrate cranially, and

(6) Widening of the disc space anteriorly with or without avulsion and/or shear chip fracture from anterior end plate.

\section{Type C}

Type $\mathrm{C}$ is a result of torsional injuries superimposed on either type A or B.

Radiographic characteristics: Features of either type A or type B along with presence of the following radiological finding suggests rotational injuries: (1) Unilateral fracture/dislocation of articular process, (2) Fracture of transverse process, (3) Asymmetrical vertebral body fracture, and (4) Lateral avulsion fracture of end plate.

\section{AO concept of stability}

(1) Type A: injuries are either stable or partially compromised but never completely unstable, as the PLC is intact. The degree of stability progresses from the most stable type A to the most unstable type A3.3, a burst fracture.

(2) In types B1 and B2: stability in flexion is always completely lost due to the PLC injury while stability in the extension is maintained by an intact ALL. B3 injuries are unstable in extension, but may be stable in flexion, if the PLC is intact. The author of the AO classification concluded that B1 \& B2 injuries may, in principle, be treated by compression of the posterior column. In B2 injuries, due to their bony nature, conservative treatment is an option, using hyperextension immobilization. But, B1 and B3 injuries, being predominantly disco-ligamentous, need surgical intervention due to their poor healing potential otherwise.

(3) Type C injuries: as a rule, all type C injuries (with rare exceptions) are unstable and should be treated surgically.

In an attempt to include all fracture patterns, the classification has a total of 53 subtypes. This complexity makes it a daunting task for any spinal surgeon to effectively use the classification in routine clinical practice. Additionally, it has demonstrated poor inter- and intra-observer reliability, with only $67 \%$ agreement when only the three major types are considered. The reliability markedly decreases when subtypes are also compared $[3,18,19]$. Furthermore, this scheme does not present a concrete 
definition of stability or include neurological deficit as a criterion. Subsequent research found little co-relation between magnetic resonance imaging (MRI) findings of instability based on PLC integrity and the AO classification [20].

\section{The Thoracolumbar Injury Severity Score and Thoracolumbar Injury Classification (2005)}

Vaccaro et al. [21-24] criticized the existing classification in three respects; first, it was too complex to use in routine clinical practice; second, it failed to recognize the importance of PLC and neurological status, and third, it did not suggest treatment options. Vaccaro et al. [21,22] considered that the morphology of an injury suggests its immediate mechanical instability, the PLC status determines long-term stability, and that the neurological status at the time of injury dictates the ultimate functional prognosis. Together, the three independent variables appropriately reflect the spine stability pattern.

Vaccaro and his associates' classification system is known as the thoraco-lumber injury classification system (TLICS) and consists of a more objective scoring system following three major independent variables:

(1) Morphology of the injury: The morphology description used was similar to the $\mathrm{AO}$ system, and is based on plain X-ray, CT scan, and MRI. Individual points were assigned as shown in Fig. 4. In the presence of multiple morphology patterns, the most severely affected vertebrae is scored. For example, a compression injury alone gets a score of 1 , while if burst component is present, the score will be 2 .

(2) Integrity of the PLC: The integrity of the PLC on a plain radiograph and CT scan is indicated by the splaying of spinous process, diastases of the facet joint, perched facets, and a sub-luxated intra-articular joint. Vaccaro et al. suggested a score of 1 if the PLC is intact and a score of 3 if it is ruptured. However, if the PLC injury is subtle, he called it indeterminate, and gave it a score of 2 .

Two MRI findings which are diagnostic of PLC disruption are the discontinuation of the black stripe representing the supra-spinous ligament on T1-weighted images and the high signal intensity of the interspinous space, representing the interspinous ligament on $\mathrm{T} 2$-weighted images. Fat-suppressed T2 images are more sensitive and specific in the evaluation of the PLC.
(3) Neurological status of the patient: Since neurological status determines overall prognosis and outcome, it was also considered in the scoring system, and scored separately depending on nerve root involvement, and incomplete or complete spinal cord involvement.

\section{Vaccaro's [21-24] Concept of Stability}

The point values of above three mentioned categories are added and the total sum reflects the injury severity score which has potential impact on mechanical or neurological stability. A score of 3 or less suggest non-operative management, while if the score is 5 or more, operative

\begin{tabular}{|c|c|c|}
\hline \multirow{3}{*}{\multicolumn{2}{|c|}{$\begin{array}{l}\text { Identify morphology } \\
\text { of injury }\end{array}$}} & \multirow{2}{*}{$\begin{array}{l}\text { Assessment - Score } \\
\text { Compression - } 1\end{array}$} \\
\hline & & \\
\hline & & Burst fracture - 2 \\
\hline & & Lateral angulation $>15^{\circ}-2$ \\
\hline & & Translation / rotation - 3 \\
\hline & & Distraction - 4 \\
\hline \multirow{3}{*}{\multicolumn{2}{|c|}{$\begin{array}{l}\text { Assess posterior } \\
\text { ligament complex } \\
\text { integrity }\end{array}$}} & Intact - 1 \\
\hline & & Ruptured - 3 \\
\hline & & In-determinant - 4 \\
\hline \multirow{5}{*}{\multicolumn{2}{|c|}{$\begin{array}{l}\text { Assess neurological } \\
\text { status }\end{array}$}} & Intact - 0 \\
\hline & & Incomplete cord - 3 \\
\hline & & Complete cord - 2 \\
\hline & & Nerve root - 2 \\
\hline & & Cauda equine - 3 \\
\hline Total sore & \multicolumn{2}{|c|}{ Proposed management guide line } \\
\hline$<3$ & \multicolumn{2}{|c|}{ Non-operative } \\
\hline 4 & \multicolumn{2}{|c|}{ Non-operative or operative } \\
\hline$>4$ & \multicolumn{2}{|c|}{ Operative } \\
\hline \multicolumn{3}{|c|}{$\begin{array}{l}\text { Operative in presence of clinical qualifier: } \\
\text { extreme kyphosis, severe lateral angulation, } \\
\text { adjacent rib fracture, soft tissue compromise, } \\
\text { inability to brace, polytrauma }\end{array}$} \\
\hline
\end{tabular}

Fig. 4. Assigning points to a fracture on the basis of three components: morphology, integrity of the posterior ligamentous complex, and neurologic status of the patient. 
intervention is recommended. Injuries assigned a score of 4 will be handled conservatively or operatively depending on what is called "clinical qualifier".

Vaccaro et al. [23] and Lee et al. [24] proposed several guidelines with respect to the surgical approach based on a few general principles which can be summarized as: (1) An incomplete neurological injury in the presence of neural compression from an anterior element requires an anterior approach, (2) PLC disruption requires a posterior approach, and (3) A combination of the above require both posterior and anterior approaches.

By incorporating posterior ligamentous integrity and neurological status, in addition to introducing a scoring system, TLICS has certainly attempted to objectively assess the severity of the injuries and thereby help in laying down treatment guideline. TLICS is a reliable system for assessing fractures of the thoracic and lumbar spine when used by experts; however, the PLC subcomponent score was the least reliable component $[25,26]$.

\section{Discussion}

The presence of more than half a dozen of classification schemes is testimony the difficulty faced by spinal surgeons while treating TL injuries. Despite tremendous advances in diagnostic technology, including high resolution CT, MRI, and electro-diagnostic testing, spinal stability is variably defined, widely interpreted, and inconsistently measured. This leads to surgical challenges in deciding between conservative and surgical fixation, posterior or anterior approaches, and long or short instrumentation. In a 2006 Cochrane review, Yi et al. [27] identified three prospective, randomized control trials with a minimum of a 2-year follow-up. They concluded that surgery does improve kyphosis; however, no difference in clinical outcomes in terms of pain, functional outcome, and return to work status were observed. The cost of operative intervention is significant compared to non-operative bracing. With regards to short or long segment fusion, it was noted that the loss of correction and implant failure is certainly higher with short form of short segment posterior instrumentation. However, long instrumentation compromises spinal motion and causes significant blood loss. Thus, the benefits must be carefully balanced with the morbidity of sacrificing the motion of the segment [28].

Some classifications are too simple and fail accurately determine whether an injury is stable or unstable, while other systems, such as the $\mathrm{AO}$ classification, are too inclusive, complex, cumbersome, and difficult to use in routine daily practice by spine surgeons. Moreover, their reproducibility is a matter of concern; the Denis and the AO system have only moderate reliability and repeatability [19]. In addition, inter-observer agreement even for the three main injury (types $\mathrm{A}, \mathrm{B}$, and $\mathrm{C}$ ) of the $\mathrm{AO}$ classification was only $67 \%$, and the reliability markedly decreased when the 53 distinct injury patterns were included [20].

TLICS necessitates use of MRI for scoring; however, the use of MRI in emergencies is neither a routine practice nor available in every center. Known contraindications and the hemodynamic instability of polytrauma victims further limits MRI use and thus is a limitation of this classification system. Additionally, if the PLC status is indeterminant, a score of 2 is given to this finding, which has a potential to tilt the decision in favor of surgery. Similarily, in the presence of spinal shock, neurological scoring is not possible and such situations re-emphasize that the spine trauma surgeon must be familiar with other classification systems and indirect radiographic parameters of an unstable spine. Lastly, Vaccaro et al. maintain that the three major determinants are fully independent. If so, rotational injury with a score of 3 , with intact PLC and intact neurology does not require surgical stabilization. Rather, the authors believe in the traditional teaching that one is a sequela to the other if the resulting force continues, and that rotational injuries are inherently unstable and often require surgey. AO classification also places them in a category more unstable than distraction injuries.

\section{Conclusions}

In the absence of a universally accepted TL trauma classification system, it is important for spine surgeons to understand the strengths and limitations of different classification systems. A thorough understanding of the AO system, TLICS, and the load-sharing classification is important to optimize treatment for thoracolumbar injuries. Many issues, such as the presence of osteoporosis, the involvement of a disc, and skip fractures remain unresolved. In such circumstances, clinical findings like a distinct palpable gap between spinous processes and an accurate assessment of neurological deficit play important roles. Furthermore, indirect radiological signs, such as facet disruption, displaced horizontal fracture of lamina, vertebral body comminution more than $75 \%$, increased 
trans-pedicular distance, spinal canal narrowing more than $50 \%$, and doubtful PLC integrity are suggestive of an unstable spine that needs a thorough evaluation and possible surgical stabilization.

\section{Conflict of Interest}

No potential conflict of interest relevant to this article was reported.

\section{References}

1. Boehler L. Die techniek der knochenbruchbehandlung im grieden und im kriege. Vienna: Verlag von Wilheim Maudrich; 1930.

2. Smith HE, Anderson DG, Vaccaro AR, et al. Anatomy, Biomechanics, and Classification of Thoracolumbar Injuries. Semin Spine Surg 2010;22:2-7.

3. Mirza SK, Mirza AJ, Chapman JR, Anderson PA. Classifications of thoracic and lumbar fractures: rationale and supporting data. J Am Acad Orthop Surg 2002;10:364-77.

4. Cotler JM, Vernace JV, Michalski JA. The use of Harrington rods in thoracolumbar fractures. Orthop Clin North Am 1986;17:87-103.

5. Ferguson RL, Allen BL Jr. A mechanistic classification of thoracolumbar spine fractures. Clin Orthop Relat Res 1984;(189):77-88.

6. Sethi MK, Schoenfeld AJ, Bono CM, Harris MB. The evolution of thoracolumbar injury classification systems. Spine J 2009;9:780-8.

7. Watson-Jones R. The results of postural reduction of fractures of the spine. J Bone Joint Surg Am 1938;20: 567-86.

8. Nicoll EA. Fractures of the dorso-lumbar spine. J Bone Joint Surg Br 1949;31:376-94.

9. Holdsworth F. Fractures, dislocations, and fracturedislocations of the spine. J Bone Joint Surg Am 1970; 52:1534-51.

10. Denis F. The three column spine and its significance in the classification of acute thoracolumbar spinal injuries. Spine (Phila Pa 1976) 1983;8:817-31.

11. Chance GQ. Note on a type of flexion fracture of the spine. Br J Radiol 1948;21:452.

12. McAfee PC, Yuan HA, Fredrickson BE, Lubicky JP. The value of computed tomography in thoracolumbar fractures: an analysis of one hundred consecutive cases and a new classification. J Bone Joint Surg Am 1983;65:461-73.

13. White AA 3rd, Panjabi MM. The basic kinematics of the human spine: a review of past and current knowledge. Spine 1978;3:12-20.

14. McCormack T, Karaikovic E, Gaines RW. The load sharing classification of spine fractures. Spine (Phila Pa 1976) 1994;19:1741-4.

15. Dai LY, Jin WJ. Interobserver and intraobserver reliability in the load sharing classification of the assessment of thoracolumbar burst fractures. Spine (Phila Pa 1976) 2005;30:354-8.

16. Scholl BM, Theiss SM, Kirkpatrick JS. Short segment fixation of thoracolumbar burst fractures. Orthopedics 2006;29:703-8.

17. Magerl F, Aebi M, Gertzbein SD, Harms J, Nazarian S. A comprehensive classification of thoracic and lumbar injuries. Eur Spine J 1994;3:184-201.

18. Bono CM, Vaccaro AR, Hurlbert RJ, et al. Validating a newly proposed classification system for thoracolumbar spine trauma: looking to the future of the thoracolumbar injury classification and severity score. J Orthop Trauma 2006;20:567-72.

19. Wood KB, Khanna G, Vaccaro AR, Arnold PM, Harris MB, Mehbod AA. Assessment of two thoracolumbar fracture classification systems as used by multiple surgeons. J Bone Joint Surg Am 2005;87:1423-9.

20. Oner F, Ramos L, Simmermacher R, et al. Classification of thoracic and lumbar spine fractures: problems of reproducibility. Eur Spine J 2002;11:235-45.

21. Vaccaro AR, Lehman RA Jr, Hurlbert RJ, et al. A new classification of thoracolumbar injuries: the importance of injury morphology, the integrity of the posterior ligamentous complex, and neurologic status. Spine (Phila Pa 1976) 2005;30:2325-33.

22. Vaccaro AR, Zeiller SC, Hulbert RJ, et al. The thoracolumbar injury severity score: a proposed treatment algorithm. J Spinal Disord Tech 2005;18:209-15.

23. Vaccaro AR, Lee JY, Schweitzer KM Jr, et al. Assessment of injury to the posterior ligamentous complex in thoracolumbar spine trauma. Spine J 2006;6:524-8.

24. Lee JY, Vaccaro AR, Lim MR, et al. Thoracolumbar injury classification and severity score: a new paradigm for the treatment of thoracolumbar spine trauma. J Orthop Sci 2005;10:671-5.

25. Lewkonia P, Paolucci EO, Thomas K. Reliability of the thoracolumbar injury classification and severity 
score and comparison with the denis classification for injury to the thoracic and lumbar spine. Spine (Phila Pa 1976) 2012;37:2161-7.

26. Lenarz CJ, Place HM, Lenke LG, Alander DH, Oliver D. Comparative reliability of 3 thoracolumbar fracture classification systems. J Spinal Disord Tech 2009; 22:422-7.

27. Yi L, Jingping B, Gele J, Baoleri X, Taixiang W. Op- erative versus non-operative treatment for thoracolumbar burst fractures without neurological deficit. Cochrane Database Syst Rev 2006;(4):Cd005079.

28. Tezeren G, Kuru I. Posterior fixation of thoracolumbar burst fracture: short-segment pedicle fixation versus long-segment instrumentation. J Spinal Disord Tech 2005; 18:485-8. 